\title{
Generalized Dynamic Reduction in Finite Element Dynamic Optimization
}

\author{
Ki-Ook Kim* and William J. Anderson $\dagger$ \\ The University of Michigan, Ann Arbor, Michigan
}

\begin{abstract}
A UTOMATED redesign for frequency changes of undamped structural systems is carried out through generalized dynamic reduction. The redesign involves a nonlinear perturbation from a baseline design. Generalized coordinates are introduced to transform the original problem into a subspace of small size. A nonlinear mathematical programming scheme is applied in the reduced subspace.
\end{abstract}

\section{Contents}

When the analysis of dynamic systems shows undesirable natural frequencies and mode shapes, it is not easy to know where and how much to modify the baseline system to achieve the desired goals. Stetson et al., , $^{2}$ developed the first-order perturbation equations and applied the approach successfully to redesign of jet engine compressor blades.

When changes become large, however, it has been found ${ }^{3}$ that the first-order perturbation equations are not sufficient, especially for significant mode shape changes. A nonlinear inverse perturbation method was developed that proved to give accurate redesign results for beam structures.

While the mathematical programming technique combined with the finite element method is a direct and effective approach, the major difficulty in large problems is the huge computing effort necessary to evaluate the functions and their gradients with respect to the free variables. The generalized dynamic reduction is a combination of Guyan's static condensation and the subspace iteration method. Dynamic equations are formulated in terms of a set of both generalized and physical degrees of freedom. Hence, the size of the dynamic system can be reduced substantially more than through Guyan's static condensation without loss of accuracy.

The general eigenvalue problem arising in discrete structural systems can be expressed in a matrix form

$$
[k][\Phi]=[m][\Phi][\lambda]
$$

If the masses and stiffnesses are changed, the frequencies and mode shapes also change. The equilibrium equation for the perturbed eigensystem is

$$
\left[k^{\prime}\right]\left[\Phi^{\prime}\right]=\left[m^{\prime}\right]\left[\Phi^{\prime}\right]\left[\lambda^{\prime}\right]
$$

The eigenvector expansion theorem shows that any displacement vector can be expressed as a linear combination

Received July 21, 1983; synoptic received Jan. 12, 1984. Copyright (C) 1984 by W. J. Anderson. Published by the American Institute of Aeronautics and Astronautics with permission. Full paper available from National Technical Information Service, Springfield, Va. 22151, at the standard price (available upon request).

${ }^{*}$ Graduate Student; presently Project Engineer, Automated Analysis Corporation, Ann Arbor, Mich.

$\dagger$ Professor, Aerospace Engineering. of the eigenvectors. If a truncated set of $q$ modes $\left[G^{*}\right]$ is used, the relation may be only approximate,

$$
\{u\} \simeq\left[G^{*}\right]\left\{u_{q}\right\}
$$

where $\left\{u_{q}\right\}$ is the vector of dynamic degrees of freedom. As in static condensation, the physical degrees of freedom can be divided into two groups,

$$
\{u\}=\left\{\begin{array}{l}
u_{o} \\
u_{t}
\end{array}\right\} \simeq\left[\begin{array}{c}
G_{o q}^{*} \\
G_{t q}^{*}
\end{array}\right]\left\{u_{q}\right\}
$$

where $\left\{u_{o}\right\}$ is the slave set to be condensed out and $\left\{u_{t}\right\}$ the master set included in the analysis.

The reduction procedure for the slave set is defined in terms of the master set and the generalized degrees of freedom,

$$
\left\{u_{0}\right\}=\left[G_{o t} G_{o q}\right]\left\{\begin{array}{l}
u_{t} \\
u_{q}
\end{array}\right\}
$$

Hence,

$$
\left[G_{o q}\right]=\left[G_{o q}^{*}\right]-\left[G_{o t}\right]\left[G_{t q}^{*}\right]
$$

For convenience, let us consider a single mode. If there are no physical degrees of freedom in the analysis set, i.e., $\left\{u_{i}\right\}=0$, the equation of motion of the perturbed system can be simplified as

$$
\left[K_{a a}^{\prime}\right]\left\{u_{q}^{\prime}\right\}=\lambda^{\prime}\left[M_{a a}^{\prime}\right]\left\{u_{q}^{\prime}\right\}
$$

where

$$
\begin{aligned}
& {\left[K_{a a}^{\prime}\right]=\left[G_{o q}^{\prime}\right]^{T}\left[k_{o o}^{\prime}\right]\left[G_{o q}^{\prime}\right]} \\
& {\left[M_{a q}^{\prime}\right]=\left[G_{o q}^{\prime}\right]^{T}\left[m_{o o}^{\prime}\right]\left[G_{o q}^{\prime}\right]} \\
& {\left[G_{o q}^{\prime}\right]=\left[G_{o q}^{* \prime}\right]}
\end{aligned}
$$

If one assumes that $\left[G_{o q}^{*}\right]$ contains the approximate eigenvectors accurately enough to represent the lowest modes of the perturbed system, it can be used in place of $\left[G_{o q}^{* \prime}\right]$ and Eqs. (8) can be written as

$$
\begin{aligned}
{\left[K_{a a}^{\prime}\right] } & =\left[G_{o q}\right]^{T}\left[k_{o o}^{\prime}\right]\left[G_{o q}\right] \\
& =\left[G_{o q}\right]^{T}\left(\left[k_{o o}\right]+\left[\Delta k_{o o}\right]\right)\left[G_{o q}\right] \\
{\left[M_{a a}^{\prime}\right] } & =\left[G_{o q}\right]^{T}\left[m_{o o}^{\prime}\right]\left[G_{o q}\right] \\
& =\left[G_{o q}\right]^{T}\left(\left[m_{o o}\right]+\left[\Delta m_{o o}\right]\right)\left[G_{o q}\right]
\end{aligned}
$$

Substituting Eqs. (9) into Eq. (7), one gets the residual error vector in terms of a ratio of thicknesses, $\alpha_{e}=h_{e}^{\prime} / h_{e}$, 


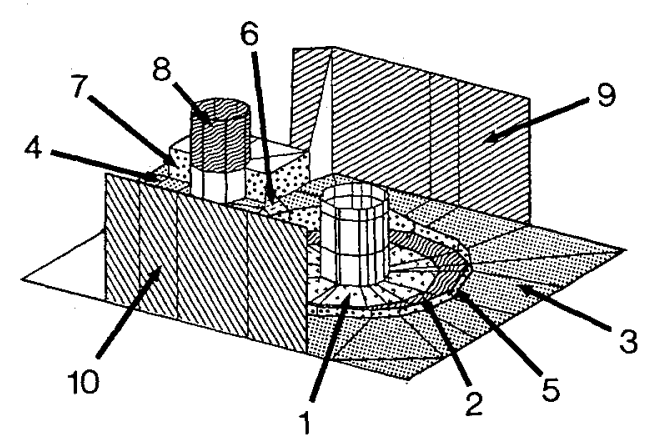

Fig. 1 Baseline system and element groups.

$$
\begin{aligned}
& \{R\} \equiv\left(\left[K_{a a}\right]-\lambda^{\prime}\left[M_{a a}\right]\right)\left\{u_{q}^{\prime}\right\}+\sum_{e=1}^{L}\left(\left[G_{o q}\right]^{T}\left[k^{S}\right]_{e}\left[G_{o q}\right]\right. \\
& \left.-\lambda^{\prime}\left[G_{o q}\right]^{T}[m]_{e}\left[G_{o q}\right]\right)\left(\alpha_{e}-1\right)\left\{u_{q}^{\prime}\right\} \\
& \quad+\sum_{e=1}^{L}\left(\left[G_{o q}\right]^{T}\left[k^{L}\right]_{e}\left[G_{o q}\right]\left(\alpha_{e}^{3}-1\right)\right)\left\{u_{q}^{\prime}\right\}=0
\end{aligned}
$$

where $\left[k^{S}\right]$ and $\left[k^{L}\right]$ are, respectively, the linear and nonlinear parts of the stiffness changes. The penalty function method $^{3}$ can be used with Eq. (10) as the equality constraint.

As a case study on a problem of practical size, redesign of a small aluminum casting ${ }^{4}$ is considered. A total of 209 grid points and 312 elements are used for the finite element modeling. Figure 1 shows the structure composed of plates and beams. The perturbed elements are divided into 10 design groups. The redesign goal will be to modify the first flexural frequency by $+30 \%$ (case 1 ) and $-10 \%$ (case 2 ).

Table 1 shows the thickness changes in each design group obtained through mathematical programming and the reanalysis results. The original objective function is the weight of the perturbed structure, which is to be minimized.

When the dynamic reduction method was applied to structural redesign for frequency changes, it was found that the frequencies obtained from the reanalysis were always lower (more negative) than the desired goal. In the subspace, however, the mathematical programming solver provided the exact desired frequency. This implies that the error is caused by the physical approximation in the truncation of the higher modes, rather than a loss of accuracy in the equation solver.

The eigenvalue problem in the subspace is

$$
\left[G_{o q}\right]^{T}\left[k_{o o}^{\prime}\right]\left[G_{o q}\right]\left\{u_{q}^{\prime}\right\}=\lambda^{\prime}\left[G_{o q}\right]^{T}\left[m_{o o}^{\prime}\right]\left[G_{o q}\right]\left\{u_{q}^{\prime}\right\}
$$

The eigenvalue problem in the original space is

$$
\left[k_{o o}^{\prime}\right]\left\{u_{o}^{\prime}\right\}=\lambda^{*}\left[m_{o o}^{\prime}\right]\left\{u_{o}^{\prime}\right\}
$$

Assume that the mode shape $\left\{u_{o}^{\prime}\right\}$ can be written as

$$
\left\{u_{o}^{\prime}\right\}=\left[G_{o q}\right]\left\{u_{q}^{\prime}\right\}+[H]\left\{u_{q}^{\prime \prime}\right\}
$$

where $[H]$ is the remaining higher part of the "approximate" eigenvectors and $\left\{u_{q}^{\prime \prime}\right\}$ contains the corresponding generalized coordinates. Hence, $[H]$ together with $\left[G_{o q}\right]$ forms an orthonormal basis of the original system $\left[m_{o o}\right]$. From Eqs. (11-13), one gets,

$$
\begin{aligned}
& \left\{u_{q}^{\prime}\right\}^{T}\left[G_{o q}\right]^{T}\left[m_{o o}^{\prime}\right]\left[G_{o q}\right]\left\{u_{q}^{\prime}\right\}\left(\lambda^{\prime}-\lambda^{*}\right) \\
& =\left\{u_{q}^{\prime}\right\}^{T}\left(\left[G_{o q}\right]^{T}\left[k_{o o}^{\prime}\right][H]-\lambda^{*}\left[G_{o q}\right]^{T}\left[m_{o o}^{\prime}\right][H]\right) \\
& \quad \times\left([H]^{T}\left[k_{o o}^{\prime}\right][H]-\lambda^{*}[H]^{T}\left[m_{o o}^{\prime}\right][H]\right)^{-I} \\
& \quad \times\left([H]^{T}\left[k_{o o}^{\prime}\right]\left[G_{o q}\right]-\lambda^{*}[H]^{T}\left[m_{o o}^{\prime}\right]\left[G_{o q}\right]\right)\left\{u_{q}^{\prime}\right\}
\end{aligned}
$$

Table 1 Determination of design variables by mathematical programming

\begin{tabular}{ccc}
\hline \multicolumn{3}{c}{ Cy mathematical programming } \\
\multicolumn{3}{c}{$\begin{array}{c}\text { Mathematical programming, } \\
\text { starting point: baseline system } \\
\text { Optimal value }\end{array}$} \\
\cline { 2 - 3 } $\begin{array}{c}\text { Design } \\
\text { variables }\end{array}$ & Case 1 & Case 2 \\
\hline$\alpha_{I}$ & 1.080372 & 0.974507 \\
$\alpha_{2}$ & 1.124226 & 0.959126 \\
$\alpha_{3}$ & 1.477911 & 0.796558 \\
$\alpha_{4}$ & 1.091535 & 0.940924 \\
$h^{\prime} / h \alpha_{5}$ & 1.456787 & 0.869299 \\
$\alpha_{6}$ & 1.455563 & 0.838210 \\
$\alpha_{7}$ & 0.926484 & 1.000864 \\
$\alpha_{8}$ & 0.917883 & 1.004945 \\
$\alpha_{9}$ & 0.949289 & 0.982071 \\
$\alpha_{10}$ & 0.989839 & 0.924348 \\
Goals & & \\
$\omega^{\prime} \times 10^{-3}$ & $3.213581(30.00 \%)$ & $2.224787(-10.00 \%)$ \\
Reanalysis & & \\
$\omega^{\prime} \times 10^{-3}$ & $3.122100(26.29 \%)$ & $2.207268(-10.71 \%)$ \\
& & \\
\hline
\end{tabular}

Since $[H]$ is associated with the higher modes, the term in the second bracket of the right-hand side is symmetric, nonsingular, and strictly diagonally dominant and hence positive definite. Therefore, the right-hand side of Eq. (14) is positive semidefinite and the exact eigenvalue $\lambda^{\prime}$ is greater than or equal to the approximate eigenvalue $\lambda^{*}$ because $\left[m_{o o}^{\prime}\right]$ is positive definite.

The inaccuracy due to the insufficiency of the subspace can be partially compensated for by scaling either the goal or the design variables. ${ }^{5}$ Also, the final design can be used as another baseline design for more design iterations.

When the redesign of mode shapes is required, static condensation might be used. A set of physical degrees of freedom is then included in the analysis, which makes the redesign problem more complicated. Work is proceeding on this topic of mode shape control in large systems.

\section{Acknowledgment}

This research was supported by NSF Research Grant MEA8019642, Inverse Perturbation in Finite Element Analysis.

\section{References}

${ }^{1}$ Stetson, K.A. and Palma, G.E., "Inversion of First-Order Perturbation Theory and its Application to Structural Design," AIAA Journal, Vol. 14, April 1976, pp. 454-460.

${ }^{2}$ Stetson, K.A. and Harrison, I.R., "A Study of Structural Redesign by Finite-Element Inverse Perturbation of Vibration Modes," United Technologies Research Center, East Hartford, Conn., Rept. R80-191942-1, Jan. 1980.

${ }^{3}$ Kim, K.O., Anderson, W.J., and Sandstrom, R.E., "Nonlinear Inverse Perturbation Method in Dynamic Analysis," AIAA Journal, Vol. 21, No. 9, Sept. 1983, pp. 1310-1316.

${ }^{4}$ Hoff, C.J., Bernitsas, M.M., and Anderson, W.J., "Nonlinear Incremental Inverse Perturbation Method for Structural Redesign," Proceedings of AIAA/ASME/ASCE/AHS SDM Conference, Lake Tahoe, Nev., May 1983, AIAA, New York, 1983, pp. 296-303.

${ }^{5}$ Kim, K.O., "Nonlinear Inverse Perturbation Method in Dynamic Redesign,' Ph.D. Dissertation, University of Michigan, Ann Arbor, Aug. 1983. 